\title{
Abundancia y diversidad larval de gasterópodos en el Caribe Mexicano en relación con la temperatura, la salinidad y el oxígeno disuelto
}

\author{
José Francisco Chávez Villegas, Martha Enríquez Díaz \& Dalila Aldana Aranda \\ Laboratorio de Biología y Cultivo de Moluscos. Centro de Investigación y de Estudios Avanzados del Instituto \\ Politécnico Nacional, Unidad Mérida, Carretera Antigua a Progreso, Km. 6, A.P. 73 Cordemex, C. P. 97310, Mérida, \\ Yucatán, México; jchavez@mda.cinvestav.mx,menriquez@mda.cinvestav.mx, daldana@mda.cinvestav.mx
}

Recibido 24-VIII-2013 Corregido 12-III-2014 Aceptado 02-IV-2014

\begin{abstract}
Abundance and larval diversity of gastropods in the Mexican Caribbean in relation with temperature, salinity and dissolve oxygen. Quintana Roo has over 500 species of gastropods but little is known of the larval stages. We sampled four sites monthly from January to December 2008 with plankton tows in triplicate and a conical net of $300 \mu \mathrm{m}$ mesh. Puerto Morelos had the highest records $\left(41.26 \pm 63.23\right.$ larvae $\left.10 \mathrm{~m}^{-3}\right)$, followed by Mahahual $\left(14.78 \pm 28.21\right.$ larvae $\left.10 \mathrm{~m}^{-3}\right)$; Sian Ka' an and Xel-Ha had the lowest densities $(9.16 \pm 16.73$ and $3.35 \pm 4.33$ larvae $\cdot 10 \mathrm{~m}^{-3}$, respectively). May and August had the highest records (33.66 \pm 67.39 and $31.78 \pm 25.92$ larvae $10 \mathrm{~m}^{-3}$, respectively), March and December the lowest $\left(1.70 \pm 1.88\right.$ and $3.18 \pm 5.27$ larvae $10 \mathrm{~m}^{-3}$, respectively). There were differences in the spatial mean density $(\mathrm{p}<0.0001)$ and time $(\mathrm{p}=0.0275)$. We identified 33 species, genera Strombus, Natica, Cerithium, Nassarius, Neritidae, Cerithiopsis, Nannodiela, Rissoina, Bulla, Bittium, Modulus and Prunum, of which 28 species were recorded in Puerto Morelos. Natica, Epithonium and Cerithium had the highest incidence in the four locations. Rev. Biol. Trop. 62 (Suppl. 3): 223-230. Epub 2014 September 01.
\end{abstract}

Key words: larvae, gastropods, composition, Mexican Caribbean.

Las larvas de organismos marinos como indicadores biológicos permiten conocer el estado de salud y potencial reproductivo de las poblaciones adultas (Scheltema, 1971; Sale et al., 2010). Quintana Roo tiene más de 500 especies de gasterópodos descritas (Vokes \& Vokes, 1983), sin embargo se sabe muy poco de las etapas larvales (de Jesús Navarrete \& Aldana Aranda, 2000).

Scheltema (1971) brinda las primeras observaciones de los factores de dispersión larval, corrientes y la identificación de diez especies de gasterópodos de aguas tropicales y templadas del Atlántico Norte. Para el Caribe Mexicano, Oliva Rivera y de Jesús Navarrete (2000) identificaron 27 especies de gasterópodos para la zona sur de Quintana Roo, México (Banco Chinchorro y Punta Gavilán) y norte de Belice (Reserva de la Biosfera de Hol-Chan).
Valtierra Vega, de Jesús Navarrete y Oliva Rivera (2000), quienes identificaron 36 especies para la costa centro del estado de Quintana Roo y por último Oliva Rivera y de Jesús Navarrete (2007) reportan 31 especies para la zona sur de Q. Roo.

Actualmente no existen estudios que incluyan al norte del Caribe Mexicano (CM) en la descripción de la composición del conjunto ("ensamble") larval de gasterópodos, por dicha razón, el objetivo principal de este estudio fue conocer la distribución, abundancia y diversidad larval de gasterópodos en el CM.

\section{MATERIALES Y MÉTODOS}

Área de estudio: Cuatro sitios ubicados a lo largo de la costa del Caribe Mexicano 
fueron seleccionados a fin de conocer la diversidad larval de gasterópodos y su variación estacional en esta región. El primero de los sitios es Puerto Morelos, cuya ubicación geográfica es $20^{\circ} 49^{\prime} 21^{\prime \prime}-20^{\circ} 51^{\prime} 21^{\prime \prime} \mathrm{N}$ y $86^{\circ} 51^{\prime} 50^{\prime \prime}$ $86^{\circ} 52^{\prime} 45^{\prime}$ 'W; el segundo es Xel-Há, localizado en las coordenadas $20^{\circ} 18^{\prime} 51^{\prime \prime}-20^{\circ} 19^{\prime} 00^{\prime \prime} \mathrm{N}$ y $87^{\circ} 21^{\prime} 20^{\prime \prime}-87^{\circ} 21^{\prime} 25^{\prime \prime} \mathrm{W}$; el tercer sitio es Sian Ka'an, localizado geográficamente entre los $19^{\circ} 44^{\prime} 28^{\prime \prime}-20^{\circ} 00^{\prime} 58^{\prime \prime} \mathrm{N}$ y $87^{\circ} 27^{\prime} 10^{\prime \prime}$ $87^{\circ} 28^{\prime} 10^{\prime \prime} \mathrm{W}$; el cuarto es Mahahual ubicado entre los $18^{\circ} 42^{\prime} 15^{\prime \prime}-18^{\circ} 42^{\prime} 57^{\prime \prime} \mathrm{N}$ y $87^{\circ} 42^{\prime} 10^{\prime \prime}$ 8742’30”W (Fig. 1).

Muestras: Se realizaron tres arrastres superficiales en cada sitio de muestreo, empleando una embarcación con motor fuera de borda a una velocidad constante de $5 \mathrm{~m} \cdot \mathrm{min}$. La duración del arrastre fue de 5 minutos a una profundidad de $1 \mathrm{~m}$, empleando una red cónica de $30 \mathrm{~cm}$ de diámetro de boca, $1.5 \mathrm{~m}$ de largo y una abertura de malla de $300 \mu \mathrm{m}$. Los muestreos se realizaron entre la línea costera y la barrera arrecifal de cada sitio con excepción de XelHá. A la par de los monitoreos, mensualmente se realizaron registros de temperatura $\left({ }^{\circ} \mathrm{C}\right)$, salinidad (ppm) y de oxígeno disuelto $\left(\mathrm{mg} \cdot \mathrm{L}^{-1}\right)$, empleando un medidor de parámetros YSI-55.
El análisis de muestras se realizó en el Laboratorio de Biología y Cultivo de Moluscos del CINVESTAV-IPN, Unidad Mérida. Las muestras fueron filtradas a $80 \mu \mathrm{m}$ a fin de eliminar el exceso de formol. Posteriormente se realizó la separación y conteo de larvas empleando un microscopio estereoscópico Leica Zoom 2000, las larvas fueron preservadas en alcohol al $70 \%$. Las larvas de gasterópodos fueron identificadas utilizando las guías de identificación de Thiriot Quievreux (1983), Davis et al. (1993) y Oliva Rivera y de Jesús Navarrete (2000 y 2007). Fueron tomadas imágenes de las larvas mediante Microscopía Electrónica de Barrido en el Laboratorio de Microscopía Electrónica del Cinvestav-IPN, Mérida.

Análisis de datos: Para estimar la densidad larval en los sitios muestreados se estandarizaron los resultados obteniendo un volumen de larvas $10 \mathrm{~m}^{-3}$, dicho resultado se obtuvo empleando la siguiente fórmula:

$$
\begin{gathered}
\text { Biomasa (larvas } \left.\cdot 10 \mathrm{~m}^{-3}\right) \text { : } \\
{\left[\mathrm{N}^{\circ} \text { Organismos / Volumen }\left(\mathrm{m}^{-3}\right)\right]^{* 10}}
\end{gathered}
$$

Se empleó el programa InfoStat Profesional/Versión 1.1 para calcular media y

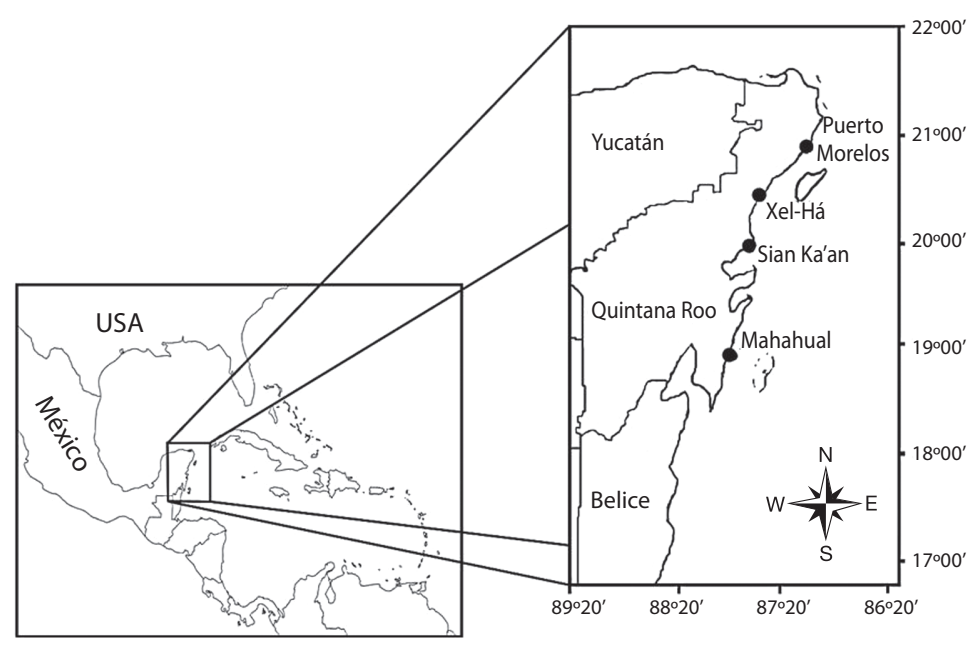

Fig. 1. Área de estudio en el Caribe Mexicano: Puerto Morelos, Xel-Há, Sian Ka'an y Mahahual. Fig. 1. Study area in the Mexican Caribbean: Puerto Morelos, Xel-Ha, Sian Ka'an and Mahahual. 
desviación estándar, análisis de varianza de una vía y prueba de comparación de medias de Tukey para la densidad larval y parámetros fisicoquímicos entre localidades y tiempo, asimismo, se realizó una correlación de Pearson entre parámetros fisicoquímicos y densidad larval.

\section{RESULTADOS}

Parámetros fisicoquímicos: El valor medio de Salinidad (ppm) fue 32.80 $\pm 4.99 \mathrm{ppm}$; en junio y septiembre se presentaron las máximas salinidades $(34.52 \pm 1.19$ y $34.50 \pm 0.87 \mathrm{ppm}$, respectivamente) y en diciembre la menor (31.24 $\pm 8.41 \mathrm{ppm})$.

La salinidad mensual por localidad fue constante en el tiempo para Mahahual, Sian Ka'an y Puerto Morelos (35.00ppm), mientras que Xel-Há presentó variación anual (32.40ppm en junio y $16.20 \mathrm{ppm}$ en diciembre). Salinidad fue significativamente diferente solo entre localidades $\left(\mathrm{p}_{(\alpha 0.05 ; \text { g.1. } 4: 44)}:<0.0001\right)$, siendo diferente Xel-Há (Tukey: $\leq 0.05$ ).

La temperatura media fue de $27.02 \pm 1.43^{\circ} \mathrm{C}$. El análisis de varianza de una vía no mostró diferencias significativas en la temperatura media entre localidades $\left(\mathrm{p}_{(\alpha 0.05 ; \text { g.l. } 4: 44)}\right.$ :
$0.2720)$, pero si en el tiempo $\left(\mathrm{p}_{(\alpha 0.05 ;}\right.$ g.l. 11:44): $0.0002)$ siendo diferente los valores registrados durante julio (Tukey: $\leq 0.05$ ).

La concentración media de oxígeno disuelto $\left(\mathrm{mg} \cdot \mathrm{L}^{-1}\right)$ fue de $5.64 \pm 1.98 \mathrm{mg} \cdot \mathrm{L}^{-1}$, con mayores registros de marzo a abril $(7.82 \pm 0.33$ y $7.68 \pm 0.38 \mathrm{mg} \cdot \mathrm{L}^{-1}$, respectivamente) y menores en octubre $\left(3.68 \pm 0.52 \mathrm{mg} \cdot \mathrm{L}^{-1}\right)$. El Oxígeno disuelto no mostró diferencias significativas entre localidades $\left(\mathrm{p}_{(\alpha 0.05 ; \text { g.1. } 4: 44)}: 0.8835\right)$, pero si en el tiempo y tiempo ( $\mathrm{p}_{(\alpha 0.05 ;}$ g.1. 11:44) $<0.0001)$ diferencias marcadas por el período marzo-mayo (Tukey : $\leq 0.05$ ).

Abundancia espacial y temporal de gasterópodos: La densidad media de larvas (larvas $\cdot 10 \mathrm{~m}^{-3}$ ) de gasterópodos para las cuatro localidades del CM varía por localidad (Fig. 2). Puerto Morelos tuvo los mayores registros (41.26 \pm 63.23 larvas $\left.\cdot 10 \mathrm{~m}^{-3}\right)$, seguido por Mahahual $\left(14.78 \pm 28.21\right.$ larvas $\left.\cdot 10 \mathrm{~m}^{-3}\right)$, mientras que Sian Ka'an y Xel-Há presentaron las menores densidades $(9.16 \pm 16.73$ y $3.35 \pm 4.33$ larvas $10 \mathrm{~m}^{-3}$, respectivamente). El análisis de varianza registró diferencias significativas en la densidad media entre localidades $\left(\mathrm{p}_{(\alpha 0.05 ; \text { g. } 1 \text {. }}\right.$ 4;167): $<0.0001)$ Siendo estadísticamente diferente la localidad de Xel-Há (Tukey $: \leq 0.05$ ).

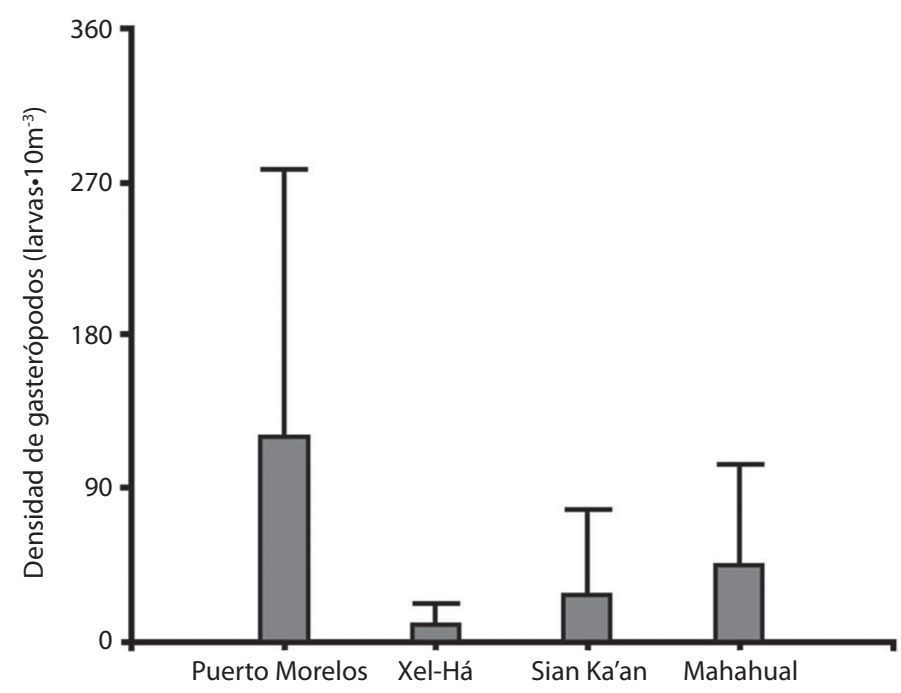

Fig. 2. Densidad media larval de gasterópodos en el Caribe Mexicano.

Fig. 2. Average larval density of gastropods in the Mexican Caribbean. 
La densidad media (larvas $10 \mathrm{~m}^{-3}$ ) de gasterópodos varia en espacio y tiempo (Fig. 3). Las mayores densidades se presentaron de junio a septiembre. En Puerto Morelos los mayores registros se observaron en abril y mayo $\left(129.35 \pm 41.83\right.$ y $150.90 \pm 76.31$ larvas $\left.\cdot 10 \mathrm{~m}^{-3}\right), \mathrm{n}$ Xel-Há durante julio y septiembre $(8.87 \pm 5.96$ y $12.98 \pm 2.45$ larvas $\cdot 10 \mathrm{~m}^{-3}$ ), para Sian $\mathrm{Ka}$ 'an las densidades fueron de $59.60 \pm 4.62$ y $13.59 \pm 11.77$ larvas $10 \mathrm{~m}^{-3}$ en agosto y septiembre, respectivamente, mientras que en Mahahual las mayores densidades se observaron en junio y agosto $\left(61.78 \pm 83.48\right.$ y $47.65 \pm 5.17$ larvas $\left.\cdot 10 \mathrm{~m}^{-3}\right)$.

El análisis de varianza mostró diferencias significativas para tres localidades $(\mathrm{p} \leq 0.0001)$ con excepción de Mahahual donde se obtuvo un valor de $\left.\mathrm{p}_{(\alpha 0.05 ; \text { g.1. 11;24) }}=0.0850\right)$. Temperatura y salinidad fueron las variables que muestran una correlación significativa, sin embargo la salinidad $(r=0.6307 ; p<0.0001)$ muestra una correlación más robusta con la disponibilidad de larvas que la temperatura $(r=0.3840 ; p=$ 0.2720) (Cuadro 1).

Diversidad estacional y espacial: El cuadro 2 y figura 4 (a, b y c) muestran la diversidad larval de gasterópodos en el CM. Se registró un total de 34 especies correspondientes a 22 géneros. La mayor diversidad de larvas se registró durante la temporada de lluvias con 25 especies, seguida de nortes (12 especies). La localidad con mayores registros de larvas fue Puerto Morelos con 28 especies, mientras que Xel-Há presentó la menor diversidad (14 especies).

Los géneros con mayor número de especies en el CM fueron Natica (con cinco especies) y Epithonium con tres especies. $23.52 \%$

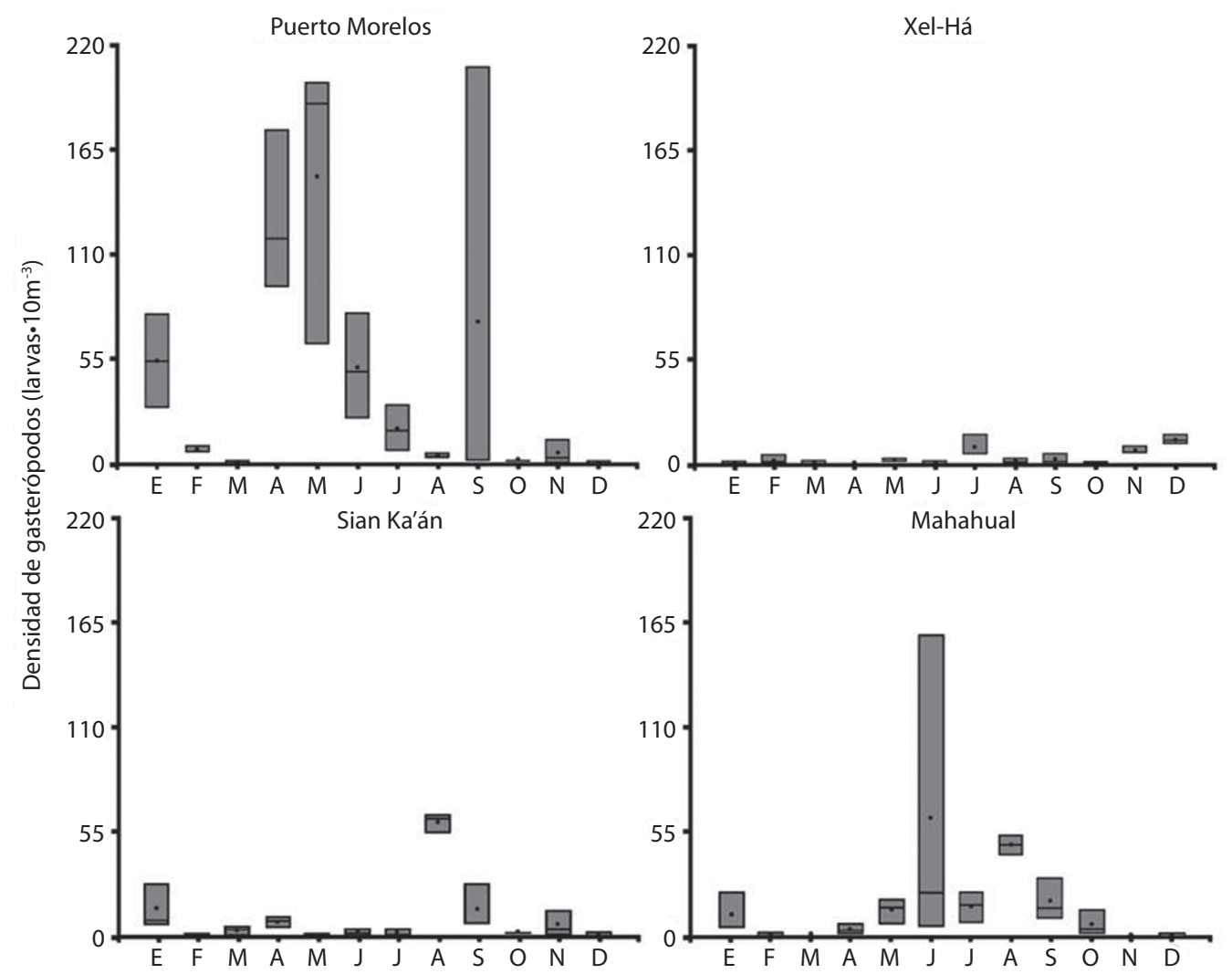

Fig. 3. Densidad media mensual de larvas de gasterópodos en el Caribe Mexicano.

Fig. 3. Monthly average density of gastropods larvae in the Mexican Caribbean. 
CUADRO 1

Medias y Análisis de correlación de Pearson (r) entre parámetros fisicoquímicos (Salinidad: ppm; temperatura: ${ }^{\circ} \mathrm{C}$; Oxígeno disuelto: $\mathrm{mg} \cdot \mathrm{L}^{-1}$ ) y densidad larval de gasterópodos (larvas $10 \mathrm{~m}^{-3}$ ) en el Caribe Mexicano

TABLE 1

Media and Pearson correlation analysis (r) among physicochemical parameters (Salinity: ppm; temperature: ${ }^{\circ} \mathrm{C}$; dissolved oxygen: $\mathrm{mg} \cdot \mathrm{L}^{-1}$ ) and gastropod larval density (larvae $10 \mathrm{~m}^{-3}$ ) in the Mexican Caribbean

\begin{tabular}{|c|c|c|c|c|c|c|c|}
\hline \multirow{2}{*}{ Parámetro } & \multicolumn{4}{|c|}{ Localidades } & \multirow{2}{*}{$\operatorname{Media}(n=48)$} & \multirow[b]{2}{*}{$\mathrm{r}$} & \multirow[b]{2}{*}{$\mathrm{p}$} \\
\hline & Puerto Morelos & Xel-Há & Sian Ka'an & Mahahual & & & \\
\hline \multirow[t]{2}{*}{ ppm } & 35.01 & 23.76 & 35 & 34.96 & 32.7 & 0.6307 & 0.000016 \\
\hline & \pm 0.05 & \pm 5.27 & \pm 0.00 & \pm 0.13 & \pm 5.00 & & \\
\hline \multirow[t]{2}{*}{${ }^{\circ} \mathrm{C}$} & 26.43 & 27.48 & 26.51 & 27.18 & 26.85 & 0.3840 & 0.2720 \\
\hline & \pm 1.59 & \pm 1.31 & \pm 1.53 & \pm 1.94 & \pm 1.60 & & \\
\hline \multirow[t]{2}{*}{$m g \cdot L^{-1}$} & 5.47 & 5.71 & 5.34 & 5.84 & 5.6 & 0.2197 & 0.0900 \\
\hline & \pm 1.48 & \pm 1.06 & \pm 1.43 & \pm 1.40 & \pm 1.34 & & \\
\hline \multirow[t]{2}{*}{ Larvas $10 \mathrm{~m}^{-3}$} & 41.26 & 3.35 & 9.16 & 14.78 & 50.56 & 1.000 & 1.000 \\
\hline & \pm 63.23 & \pm 4.33 & \pm 16.73 & \pm 28.21 & \pm 90.05 & & \\
\hline
\end{tabular}

de las especies registradas se distribuyen de Norte a Sur en las cuatro localidades de estudio, mientras que sólo $12.50 \%$ se encuentra solo en la zona Norte.

\section{DISCUSIÓN}

Stoner, Sandt y Boidron Metairon (1992) y de Jesús Navarrete (1999) señalan que la temperatura se asocia a la abundancia larval de gasterópodos, sin embargo de Jesús Navarrete (1999) no encontró una relación ente la abundancia larval y parámetros fisicoquímicos. Pacheco Archundia (2007) y Chávez Villegas (2011) señalan que la salinidad (ppm) influye en la distribución y densidad larval de gasterópodos, concretamente de $S$. gigas, lo cual concuerda con lo registrado en el presente estudio donde la salinidad se asoció en un $63 \%$ con la presencia de larvas en el CM.

La comparación de la diversidad planctónica de gasterópodos en la zona resulta difícil, debido a la escasez de trabajos realizados. En el sur del CM Oliva Rivera y de Jesús Navarrete (2000) registraron 27 especies, Valtierra Vega et al. (2000) reportan 36 especies y Oliva Rivera y de Jesús Navarrete (2007) registraron 31 especies en la zona sur de Quintana Roo y norte de Belice. Chávez Villegas (2011) señala la presencia de 32 especies de larvas de gasterópodos en el Sistema Arrecifal Mesoamericano, sin embargo no se realiza una identificación a nivel especie de estos organismos.

Actualmente no existen estudios de la diversidad larval de gasterópodos para Puerto Morelos, Sian Ka'an y Xel-Há. Oliva Rivera y de Jesús Navarrete (2000) reportan 11 especies para la costa sur de Quintana Roo (región cercana a Mahahual), de las cuales cinco especies corresponden a las 19 registradas en esta zona (Rissoina sp., Natica sp., Mitrella sp., Epitonium sp. y Cerithium sp.), asimismo, estos autores reportan una mayor abundancia y diversidad de larvas en la temporada de secas (mayo-junio), lo cual difiere a lo observado en este estudio, donde se registró una mayor diversidad en la temporada de lluvias (julio-octubre).

La mayor presencia y diversidad de larvas en el norte del CM (Puerto Morelos) podría atribuirse al efecto ascendente de las corrientes por el canal de Yucatán, sin embargo Mariño Tapia et al. (2011) señalan un fuerte flujo de corrientes internas en la laguna de Puerto Morelos, lo cual se traduce en una circulación interna de nutrientes y larvas, asimismo Coronado et al. (2007) mencionan que la tasa de intercambio entre la laguna y el océano es baja, 
CUADRO 2

Diversidad larval de gasterópodos por Temporada (Secas, lluvias y nortes) y localidades en el caribe Mexicano

TABLE 2

Diversity larval gastropods by season (Dry, rain and north wind) and localities in the Mexican Caribbean

\begin{tabular}{|c|c|c|c|c|c|c|c|}
\hline \multirow[b]{2}{*}{ Especie } & \multicolumn{3}{|c|}{ Temporada } & \multicolumn{4}{|c|}{ Localidades } \\
\hline & $\begin{array}{c}\text { Secas } \\
\text { (Mar-Jun) }\end{array}$ & $\begin{array}{l}\text { Lluvias } \\
\text { (Jul-Oct) }\end{array}$ & $\begin{array}{c}\text { Nortes } \\
\text { (Nov-Feb) }\end{array}$ & $\begin{array}{c}\text { Puerto } \\
\text { Morelos }\end{array}$ & Xel-Há & Sian Ka'an & Mahahual \\
\hline Alaba incerta & & $\mathrm{X}$ & & $\mathrm{X}$ & $\mathrm{X}$ & & $\mathrm{X}$ \\
\hline Bittium sp. 1 & & $\mathrm{X}$ & & $\mathrm{X}$ & & & \\
\hline Bittium sp. 2 & & $\mathrm{X}$ & & & $\mathrm{X}$ & $\mathrm{X}$ & $\mathrm{X}$ \\
\hline Bulla striata & & $\mathrm{X}$ & & $\mathrm{X}$ & & & \\
\hline Bulla sp. & & $\mathrm{X}$ & & & $\mathrm{X}$ & $\mathrm{X}$ & $\mathrm{X}$ \\
\hline Cerithiopsis hero & & & $\mathrm{X}$ & $\mathrm{X}$ & $\mathrm{X}$ & $\mathrm{X}$ & $\mathrm{X}$ \\
\hline Cerithium atratum & & $\mathrm{X}$ & & $\mathrm{X}$ & & & \\
\hline Cerithium sp. & & $\mathrm{X}$ & & $\mathrm{X}$ & $\mathrm{X}$ & $\mathrm{X}$ & $\mathrm{X}$ \\
\hline Choristes tenera & $\mathrm{X}$ & & & $\mathrm{X}$ & & $\mathrm{X}$ & \\
\hline Cyclostremiscus schrammii & & $\mathrm{X}$ & $\mathrm{X}$ & $\mathrm{X}$ & & & \\
\hline Epithonium albidum & & $\mathrm{X}$ & & $\mathrm{X}$ & $\mathrm{X}$ & $\mathrm{X}$ & $\mathrm{X}$ \\
\hline Epithonium krebssi & $\mathrm{X}$ & $\mathrm{X}$ & $\mathrm{X}$ & $\mathrm{X}$ & & $\mathrm{X}$ & \\
\hline Epithonium lamellosum & & $\mathrm{X}$ & & & $\mathrm{X}$ & & \\
\hline Euchelus guttarosea & & & $\mathrm{X}$ & $X$ & & & \\
\hline Mitrella lunata & & $\mathrm{X}$ & & $\mathrm{X}$ & & $\mathrm{X}$ & \\
\hline Modulus modulus & $\mathrm{X}$ & $\mathrm{X}$ & & $\mathrm{X}$ & & $\mathrm{X}$ & \\
\hline Nassarius albus & & $\mathrm{X}$ & $\mathrm{X}$ & $\mathrm{X}$ & & $\mathrm{X}$ & $\mathrm{X}$ \\
\hline Nassarius sp. & & $\mathrm{X}$ & & $\mathrm{X}$ & $\mathrm{X}$ & $\mathrm{X}$ & $\mathrm{X}$ \\
\hline Natica canrena & & & $\mathrm{X}$ & $\mathrm{X}$ & & & $\mathrm{X}$ \\
\hline Natica cayenensis & $\mathrm{X}$ & & & $\mathrm{X}$ & $\mathrm{X}$ & & \\
\hline Natica lívida & & $\mathrm{X}$ & & $\mathrm{X}$ & & & \\
\hline Natica pusilla & $\mathrm{X}$ & $\mathrm{X}$ & $\mathrm{X}$ & $\mathrm{X}$ & & & $\mathrm{X}$ \\
\hline Natica sp. & $\mathrm{X}$ & & & $X$ & $\mathrm{X}$ & $\mathrm{X}$ & $\mathrm{X}$ \\
\hline Nitidella nítida & & $\mathrm{X}$ & & $\mathrm{X}$ & & & \\
\hline Odostomia solidula & & $\mathrm{X}$ & & & & $\mathrm{X}$ & $\mathrm{X}$ \\
\hline Parviturboides interruptus & & $\mathrm{X}$ & & & & $\mathrm{X}$ & \\
\hline Pseudostomatella coccinea & & & $\mathrm{X}$ & $\mathrm{X}$ & & $\mathrm{X}$ & $\mathrm{X}$ \\
\hline Rissoina multicostata & & $\mathrm{X}$ & & $\mathrm{X}$ & & & \\
\hline Rissoina $\mathrm{sp}$. & & $\mathrm{X}$ & $\mathrm{X}$ & $\mathrm{X}$ & $\mathrm{X}$ & $\mathrm{X}$ & $\mathrm{X}$ \\
\hline Sinum maculatum & & $\mathrm{X}$ & $\mathrm{X}$ & $\mathrm{X}$ & $\mathrm{X}$ & $\mathrm{X}$ & $X$ \\
\hline Sinum perspectivum & & $\mathrm{X}$ & $\mathrm{X}$ & $\mathrm{X}$ & & $\mathrm{X}$ & $\mathrm{X}$ \\
\hline Teignostoma megastoma & & $\mathrm{X}$ & & & $\mathrm{X}$ & $X$ & $X$ \\
\hline Triphora nigrocincta & & & $\mathrm{X}$ & $\mathrm{X}$ & $\mathrm{X}$ & $\mathrm{X}$ & $\mathrm{X}$ \\
\hline Strombus gigas & $\mathrm{X}$ & $\mathrm{X}$ & & $\mathrm{X}$ & & $\mathrm{X}$ & $X$ \\
\hline$n$ & 7 & 25 & 12 & 28 & 14 & 21 & 19 \\
\hline
\end{tabular}

lo cual sugiere que los organismos encontrados en la región provienen de la población de adultos de la localidad.

Con este trabajo se asume que los sitios estudiados en el CM, especialmente los situados en la zona norte, actúan como fuente de larvas de gasterópodos, siendo sitios importantes para preservar la diversidad larval en la región.

Con base en los resultados obtenidos podemos determinar que existe un significativo 


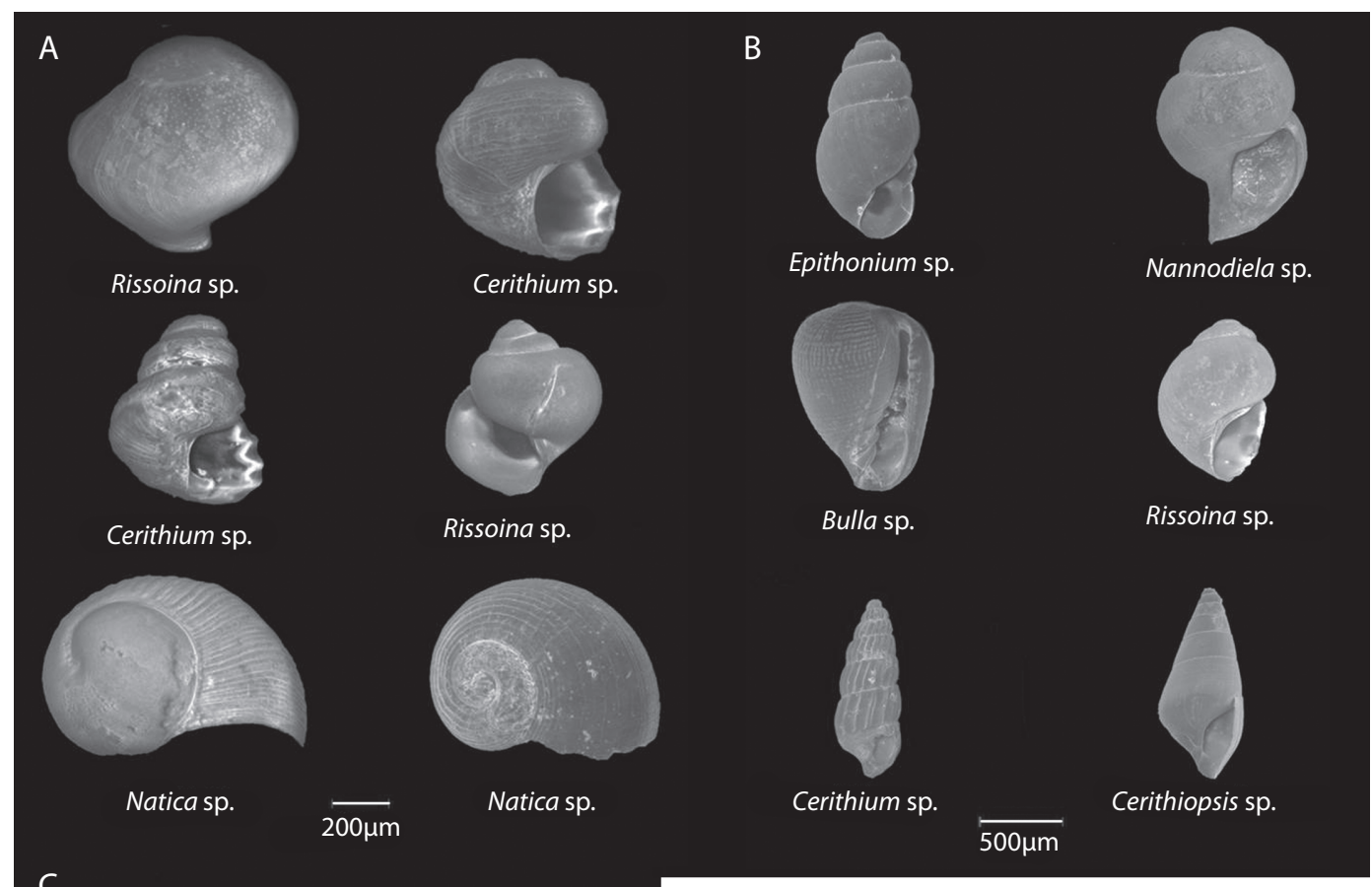

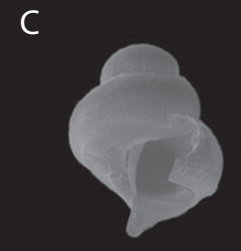

Strombus gigas

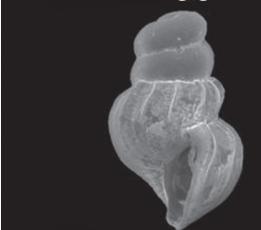

Cerithium atratum

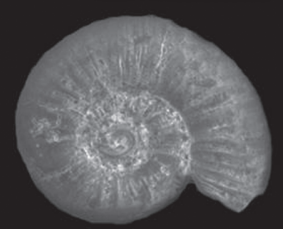

Natica sp.

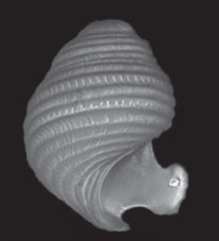

Bittium sp.

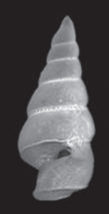

Cerithiopsis sp.

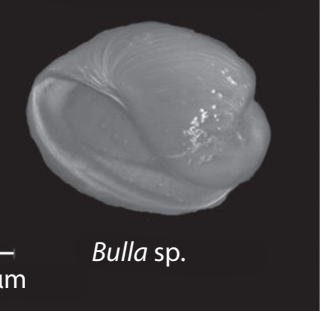

efecto de la salinidad sobre la diversidad larval de gasterópodos en el Caribe Mexicano determinado por la presencia de lluvias, época en la que se presenta la mayor diversidad y abundancia larval. Se observó un gradiente (Norte-Sur)
Fig. 4. A, B and C. Diversidad larval en el Caribe Mexicano.

Fig. 4. A, B and C. Larval diversity in the Mexican Caribbean.

en la abundancia y diversidad larval, por lo cual se considera a Puerto Morelos como un importante sitio para la conservación de la biodiversidad en el Caribe Mexicano.

\section{AGRADECIMIENTOS}

Al Centro de Investigación y de Estudios Avanzados del Instituto Politécnico Nacional, al parque acuático Xel-Há y a la Comisión Nacional de Áreas Naturales Protegidas CONANP-Puerto Morelos por las facilidades para realizar este proyecto. Al Consejo Nacional de Ciencia y Tecnología (Conacyt) por financiar este proyecto y por la beca $\mathrm{N}^{\circ} 240157$ otorgada a JF Chávez Villegas y al proyecto "Variación espacio temporal del patrón reproductivo del caracol rosado Strombus gigas en diferentes hábitats y su modelo biofísico de 
conectividad para el Caribe" (Clave: 50094) por financiar esta investigación. A Dora Huerta y Ana Cristóbal del Laboratorio de Microscopia Electrónica del Cinvestav-IPN por su apoyo en la toma de imágenes.

\section{RESUMEN}

Quintana Roo tiene mas de 500 especies de gasterópodos pero muy poco se conoce de los estadios larvales. Muestreamos cuatro sitios mensualmente de enero a diciembre de 2008 con arrastres de plancton por triplicado y con una red cónica con malla de $300 \mu \mathrm{m}$. Puerto Morelos tuvo los mayores registros $\left(41.26 \pm 63.23\right.$ larvas $\left.\cdot 10 \mathrm{~m}^{-3}\right)$, seguido por Mahahual $\left(14.78 \pm 28.21\right.$ larvas $\left.\cdot 10 \mathrm{~m}^{-3}\right)$, Sian Ka'an y Xel-Há presentaron las densidades menores $\left(9.16 \pm 16.73\right.$ y $3.35 \pm 4.33$ larvas $\cdot 10 \mathrm{~m}^{-3}$, respectivamente). Mayo y agosto presentaron los mayores registros (33.66 \pm 67.39 y $31.78 \pm 25.92$ larvas $\cdot 10 \mathrm{~m}^{-3}$, respectivamente), marzo y diciembre los menores $(1.70 \pm 1.88$ y $3.18 \pm 5.27$ larvas $10 \mathrm{~m}^{-3}$ respectivamente). Se registraron diferencias significativas en la densidad media espacial $(\mathrm{p}:<0.0001)$ y temporal (p: 0.0275). Identificamos un total de 33 especies de gasterópodos de los géneros Strombus, Natica, Cerithium, Nassarius, Neritidae, Cerithiopsis, Nannodiela, Rissoina, Bulla, Bittium, Modulus y Prunum, de las cuales 28 especies se registraron en Puerto Morelos. Natica, Epithonium y Cerithium fueron los géneros con mayor incidencia en las cuatro localidades.

Palabras clave: Larvas, gasterópodos, composición, Caribe Mexicano.

\section{REFERENCIAS}

Chávez Villegas, J. F. (2011). Abundancia espacio-temporal de larvas de Strombus gigas (Linnaeus, 1758) en el Sistema Arrecifal Mesoamericano. Tesis de maestría, Centro de Investigación y de Estudios Avanzados del Instituto Politécnico Nacional.

Coronado, C., Candela, J., Iglesias Prieto, R., Sheinbaum, J., López, M., \& Ocampo Torres, F. J. (2007). On the circulation in the Puerto Morelos fringing reef lagoon. Coral Reefs, 26, 149-163.

Davis, M., Bolton, C. A., \& Stoner, A. W. (1993). A comparison of larval development, growth, and shell morphology in three Caribbean Strombus species. Veliger, 36, 236-244.

de Jesús Navarrete, A. (1999). Distribución y abundancia de larvas velígeras de Strombus gigas en Banco Chinchorro Quintana Roo, México. Tesis doctoral, Centro de Investigación y de Estudios Avanzados del Instituto Politécnico Nacional. de Jesús Navarrete, A., \& Aldana Aranda, D. (2000). Distribution and abundance of Strombus gigas veligers at six fishing sites on Banco Chinchorro, Quintana Roo, Mexico. Journal of Shellfish Research, 19(2), 891-895.

Mariño Tapia, I., Silva Casarín, R., Enríquez Ortiz, C., Mendoza Baldwin, E., Escalante Mancera, E., \& Ruiz Rentería, F. (2010). Wave transformation and wave-driven circulation on natural reefs under extreme hurricane conditions. Coastal Engineering, 32, 28(1-9).

Oliva Rivera, J. J., \& de Jesús Navarrete, A. (2000). Composición, distribución y abundancia de larvas de moluscos gasterópodos en el sur de Quintana Roo, México y Norte de Belice. Revista de Biologia Tropical, 48(4), 77-83.

Oliva Rivera, J. J., \& de Jesús Navarrete, A. (2007). Larvas de moluscos gasterópodos del sur de Quintana Roo, México. Hidrobiológica, 17(2), 151-158.

Pacheco Archundia, V. (2007). Abundancia de larvas de Caracol rosa Strombus gigas en el área de Xcaret y Xel-Há, Quintana Roo, México. Tesis de Licenciatura, Técnico de Estudios Superiores de Huixquilucán.

Sale, P. F., Van Lavieren, H., Ablan Lagman, M. C., Atema, J., Butler, M., Fauvelot, C., Hogan, J. D., Jones, G. P., Lindeman, K. C., Paris, C. B., Steneck, R., \& Stewart, H. L. (2010). Conservando la Conectividad de los Arrecifes: Guía Para los Administradores de las Áreas Marinas Protegidas. Grupo de Trabajo de Conectividad, Programa de Investigación Dirigido a los Arrecifes de Coral y a la Creación de Capacidades para la Gestión, UNU-INWEH.

Scheltema, R. S. (1971). Larval dispersal as a means of genetic Exchange betwen geographically separated populations of shallow- wáter benthic marine gasteropods. Biological Bulletin, 140, 284-322.

Stoner, A. W., Sandt, V. J., \& Boidron Metairon, I. F. (1992). Seasonality in reproductive activity and larval abundance of queen conch, Strombus gigas. Fishery Bulletin, 90, 161-170

Thiriot Quiévreux, C., \& Scheltema, R. S. (1982). Planktonic larvae of New England gastropods. V. Bittium alternatum, Thriphora nigrocincta, Cerithiopsis emersoni, Lunatia heros and Crepidula plana. Malacologia, 23(1), 37-46.

Valtierra Vega, M. T., de Jesús Navarrete, A., \& Oliva Rivera, J. J. (2000). Análisis de la Composición, Distribución y Abundancia de larvas de Moluscos Gasterópodos, en la costa sur de Quintana Roo. México. Universidad y Ciencia, 16(32), 27-34.

Vokes, H. B., \& Vokes, E. H. (1983). Distribution of Shallow-Water marine mollusca, Yucatán Peninsula, México. Middle American Research Institute, 54, $1-183$. 